\title{
La Declaración de Cartagena desde la interdisciplinariedad
}

\author{
The Cartagena Declaration from interdisciplinarity \\ A Declaração de Cartagena desde a interdisciplinariedade
}

\author{
Sonia Echeverri ${ }^{1 *}$
}

Recibido: 1 de noviembre de 2019. Aceptado para publicación: 13 de noviembre 2019. https://doi.org/10.35454/rncm.v2supl1.031

\section{Resumen}

La Federación Latinoamericana de Terapia Nutricional, Nutrición Clínica y Metabolismo (FELANPE), en su asamblea general realizada en el pasado mes de mayo en la ciudad de Cartagena, elevó a la categoría de derecho humano el derecho al cuidado nutricional y a la lucha contra la malnutrición. La Declaración de Cartagena, la cual a través de 13 Principios aborda el preocupante problema de la desnutrición, en todas sus formas, indica la manera de crear conciencia sobre este flagelo del que mucho se habla, se investiga, se asume, se palia, se obtienen escasos resultados, pero al que pocas veces se consigue vencer.

Se reconoce, entonces, que la malnutrición es un problema complejo, que requiere tratamientos complejos, los cuales logran ser optimizados cuando disciplinas relacionadas con la actividad nutricional como medicina, enfermería, nutrición, farmacia y rehabilitación, entre otras, deciden sumar esfuerzos basados en sus conocimientos y respetando las competencias disciplinares, como quedó explícito en el Principio \# 4 de la Declaración de Cartagena enfocado en la interdisciplinariedad del cuidado nutricional, y como lo reflejan Humberto Arenas, Alexandra Texeira, Gil Hardy, María Isabel Pedreira, autores que integran este artículo que trata sobre dicho Principio.
Summary

The Latin American Federation of Nutrition Therapy, Clinical Nutrition and Metabolism (FELANPE), in its general assembly held last May in the city of Cartagena, raised the right to nutritional care and the fight against malnutrition to the category of human right. The Cartagena Declaration, which through 13 Principles addresses the worrying problem of malnutrition, in all its forms, indicates how to raise awareness about this scourge that is much talked about, investigated, assumed, palliated, they obtain few results, but which rarely wins.

It is recognized, then, that malnutrition is a complex problem, requiring complex treatments, which are optimized when disciplines related to nutritional activity such as medicine, nursing, nutrition, pharmacy and rehabilitation, among others, decide to join efforts based on their knowledge and respecting the disciplinary competences, as was explicit in Principle \# 4 of the Cartagena Declaration focused on the interdisciplinarity of nutritional care, and as reflected by Humberto Arenas, Alexandra Texeira, Gil Hardy, María Isabel Pedreira, authors who integrate this article which is about this Principle.

\section{Resumo}

A Federação Latino-Americana de Terapia Nutricional, Nutrição Clínica e Metabolismo (FELANPE), em sua assembleia geral realizada em maio passado na cidade de Cartagena, elevou o direito ao cuidado nutricional e o combate à desnutrição à categoria de direito humano. La Declaração de Cartagena, que através de 13 princípios aborda o preocupante problema da desnutrição, em todas suas formas, indica como aumentar a conscientização sobre esse flagelo que é muito comentado, investigado, assumido, aliviado, com poucos resultados, mas raramente é abatido.

Reconhece-se, então, que a desnutrição é um problema complexo, que requer tratamentos complexos, otimizados quando disciplinas relacionadas à atividade nutricional, como medicina, enfermagem, nutrição, farmácia e reabilitação, entre outras, decidem unir esforços com base em seus conhecimentos. e respeitando as competências disciplinares, como foi explicitado no Princípio no 4 da Declaração de Cartagena, focado na interdisciplinaridade do cuidado nutricional, e refletido por Humberto Arenas, Alexandra Texeira, Gil Hardy, María Isabel Pedreira, autores que integram este artigo que trata deste Princípio.

\footnotetext{
Comité de Ética Hospitalaria y de Humanismo y Bioética. Fundación Santa Fe de Bogotá, Bogotá, D.C., Colombia. FASPEN. Vice-presidente Región Centro 2019-2020.
} 
En conclusión, al firmar la Declaración de Cartagena, las sociedades, asociaciones y colegios que conforman la FELANPE, se comprometieron a reconocer la importancia de promover la integración disciplinar equitativa para brindar un óptimo cuidado nutricional, tanto en la asistencia como en la academia, según lo demuestra la evidencia científica.

Palabras clave: Declaración de Cartagena, desnutrición, interdisciplinariedad, principios.
In conclusion, by signing the Cartagena Declaration, the societies, associations and schools that make up FELANPE, pledged to recognize the importance of promoting equitable disciplinary integration to provide optimal nutritional care, both in attendance and in academia, according to It is evidenced by scientific evidence.

Keywords: Cartagena Declaration; Malnutrition; Interdisciplinary; Principles.
Concluindo, ao assinar a Declaração de Cartagena, as sociedades, associações e escolas que compõem a FELANPE comprometeram-se a reconhecer a importância de promover uma integração disciplinar equitativa para oferecer um atendimento nutricional ideal, tanto no atendimento quanto na academia, de acordo com evidências científicas.

Palavras-chave: Declaração de Cartagena, desnutrição, interdisciplinaridade, princípios.

\section{INTRODUCCIÓN}

El Principio \# 4 de la Declaración de Cartagena con acierto orienta el cuidado nutricional desde la interdisciplinariedad: "Este enfoque implica la integración equitativa de las diversas disciplinas relacionadas con la actividad nutricional. La evidencia científica muestra las ventajas de este enfoque en términos de costoefectividad, seguridad, eficiencia y eficacia." ${ }^{(1)}$

La desnutrición, en todas sus formas, es una alteración con un elevado grado de complejidad y de incertidumbre, y como tal se debe enfrentar. Esta complejidad hace que sea necesaria la participación de diversas disciplinas que enfoquen su mirada y acción a interactuar entre sí compartiendo saberes, experiencias y objetivos.

Desde hace más de cuatro décadas, cuando el doctor Stanley J. Dudrick, utilizó la nutrición parenteral con los resultados conocidos, lo hizo con el concurso de un equipo de salud conformado por profesionales de enfermería, farmacia y nutrición, inicialmente multidisciplinario fue migrando a la interdisciplinariedad. Se reconoce, desde entonces, que el aporte de cada disciplina es en gran medida responsable del éxito de la terapia nutricional: salvar vidas, lograr que pacientes condenados a morir por la imposibilidad de usar el tracto digestivo puedan nutrirse, recuperarse, salir adelante.

\section{CUIDADO NUTRICIONAL INTERDISCIPLINARIO}

Interdisciplinario, término acuñado por el sociólogo Louis Wirtz y publicado por primera vez en 1937. La interdisciplinariedad, cuyo prefijo inter, anticipa que entre las disciplinas se establecerá una relación en la que cada una aportará dentro de su campo y competen- cia, los diferentes esquemas conceptuales de análisis, sometiéndolas a comparación, juicio crítico y, finalmente, incorporando e integrando resultados con el fin de resolver un problema concreto en toda su complejidad, en este caso la desnutrición, objeto de estudio y cuidado(2).

Como lo plantea Mario Tamayo y Tamayo, la interdisciplinariedad es una exigencia interna de las ciencias. Tomemos el caso de medicina, enfermería, nutrición, farmacia, rehabilitación, psicología, cada una de ellas analizadas en particular, puede ser considerada un nivel de ciencia, que tiene como objeto mantener la salud, prevenir, tratar, curar o paliar enfermedades, entre otros, aplica procesos estructurados, que responden a una dinámica propia y que se va desarrollando a medida que cada sistema, de su propia disciplina, se conecta, se relaciona y coexiste con las otras disciplinas ${ }^{(2)}$.

Por lo tanto, enfrentar el flagelo de la malnutrición (desnutrición u obesidad), fenómeno amplio y complejo, requiere el concurso de diversas disciplinas de la salud, cada una de ellas con competencias determinadas en su contexto curricular y con una estructura o base común cuya realidad se define, estudia, investiga y desarrolla desde una visión propia. Con este fundamento (para el tema que nos ocupa, en la investigación y en la asistencia), las disciplinas se van entrelazando, integrando, dándose una cohesión de saberes, formando grupos o equipos, que enriquecen a cada disciplina, en lo personal, en lo profesional, optimizando la atención nutricional que se brinda al paciente, familia, cuidadores, y comunidad.

Sin embargo, las disciplinas adoptan diversas formas de coexistir, de trabajar en equipo, de producir conocimiento, como son la multidisciplinariedad, pluridisciplinariedad, interdisciplinariedad, intradisciplinariedad 
y la transdisciplinariedad, una de ellas con características propias, dinámicas específicas y diferentes matices. En general, los equipos de Soporte Nutricional (Nutritional Support Team, NTS, por su sigla en inglés) acogen la multidisciplinariedad: "conjunto de disciplinas con objetivos comunes que no mantienen relaciones de colaboración entre sí».

Algunos estudiosos de temas económicos relacionados con políticas de salud, proponen que la transdisciplinariedad, definida esta como "la transformación e integración del conocimiento desde todas las perspectivas interesadas para definir y tratar problemas complejos" o "Proceso según el cual los límites de las disciplinas individuales se trascienden para tratar problemas desde perspectivas múltiples con vista a generar conocimiento emergente.", podría incrementar los beneficios en términos financieros ${ }^{(1)}$.

La Declaración de Cartagena, aborda el tema del cuidado nutricional desde la Interdisciplinariedad por todas las ventajas que trae consigo, entre ellas su esencia: es la sumatoria de conocimientos, metódos científicos, habilidades y destrezas disciplinares que permiten resolver problemas complejos que sobrepasan los límites y competencias de cada disciplina, creando nuevos conocimientos, encontrando certezas en situaciones de incertidumbre, complementando deficiencias con el objetivo de optimizar el cuidado nutricional y luchar contra la desnutrición, contribuir al bienestar de la sociedad y por ende al progreso económico ${ }^{(1,3)}$.

En consecuencia, reconocer la importancia del enfoque interdisciplinario en el cuidado nutricional tiene implicaciones principalmente académicas, pero también sobre organizaciones e instituciones de salud. La administración de la terapia nutricional deberá administrarse en el marco de equipos de terapia nutricional, donde participen como mínimo, profesionales de la nutrición, enfermería, medicina, farmacia. Además, se aconseja la inclusión de otras disciplinas como la fonoaudiología, la terapia ocupacional, la fisioterapia, la rehabilitación, el trabajo social y la psicología que incrementan la eficacia de la terapia nutricional, se complementen, pero sobre todo que esta se haga con ética, humanismo, reconociendo al otro como par, respetando las diferencias, la diversidad, que el cuidado nutricional se brinde valorando y apreciando la vulnerabilidad del enfermo y la dignidad humana (Figura 1).

El doctor Óscar Jaramillo, en 1999, en comunicación a la FELANPE, en respaldo a la creación del Curso Interdisciplinario de Nutrición Clínica, CINC, escribió: "Los problemas que deben resolverse día a día, no se presentan - para nuestra fortuna o nuestra desgraciaclasificados en bloques disciplinarios. La naturaleza, en su admirable sabiduría, no permite - con muy escasas excepciones- que uno solo de sus individuos sea responsable de cambiar su curso". "... Se pretende que de una educación no interdisciplinaria dada a los estudiantes, se origine -por generación espontánea- un trabajo interdisciplinario, olvidando que la formación universitaria raras veces ofrece la oportunidad a sus estudiantes de interactuar con otras disciplinas". En este sentido la Declaración de Cartagena, recomienda que: "Desde lo académico, no solo se debe formar a los profesionales de estas disciplinas en el campo de la nutrición clínica, sino definir y respetar las competencias disciplinares específicas."

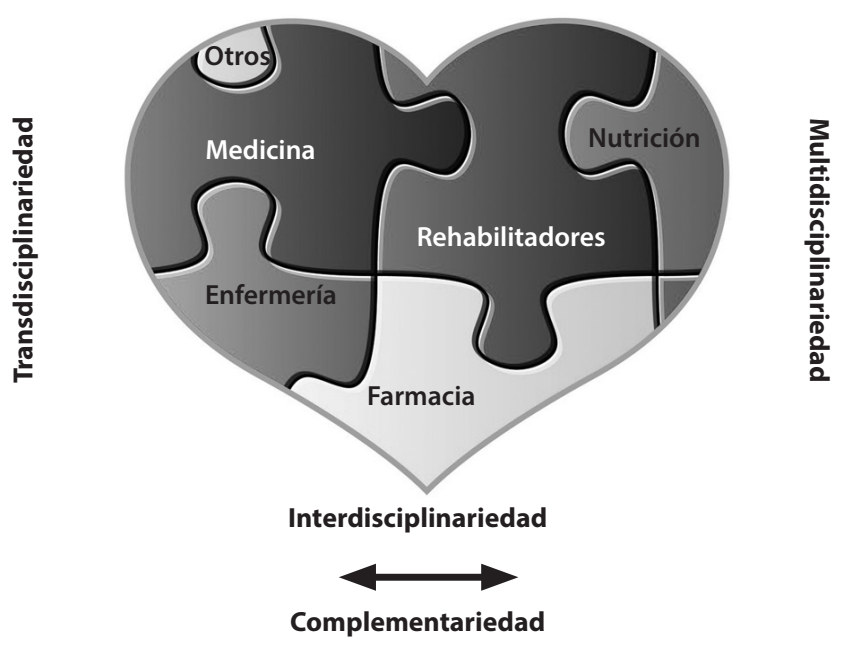

Figura 1. Modelos de trabajo en equipo para el cuidado nutricional. 


\title{
Importancia de la Declaración de Cartagena sobre el derecho al cuidado nutricional y la lucha contra la malnutrición. Desde la perspectiva médica
}

\author{
Importance of the Cartagena Declaration on the right to nutritional care and \\ the fight against malnutrition. From the medical perspective \\ Importância da Declaração de Cartagena sob o direito ao cuidado nutricional e a luta \\ contra a desnutrição. Do ponto de vista médico
}

Acad. Dr. Humberto Arenas Márquez, FACS-FASPEN ${ }^{1 *}$

Las evidencias demuestran que la tasa de prevalencia de malnutrición hospitalaria es alarmante y que los costos de atención asociados a ella se incrementan de forma exponencial ${ }^{(4,5)}$; sin duda, la nutrición óptima está íntimamente relacionada con el resultado positivo del manejo de una enfermedad ${ }^{(6)}$; por tanto, lo ideal sería que un plan nutricional se aplicara a todo enfermo que ingrese a un hospital ${ }^{(7,8)}$. Sin embargo, la realidad es distinta; el médico que lidera el equipo de atención no ha sido entrenado para afrontar este reto y lo que es peor, no está consciente de esta necesidad.

Basado en el principio hipocrático ético "Primero no dañar", la FELANPE ha presentado la Declaración de Cartagena en 2019 y definido 13 Principios para concientizar especialmente a los médicos y a los líderes de los Sistemas de Salud en Latinoamérica acerca del derecho que tienen los pacientes a una nutrición intrahospitalaria óptima a través de la creación de una Cultura Institucional que valore el cuidado nutricional ${ }^{(9)}$.

El médico como líder del equipo interdisciplinario de salud por medio de su actitud y valiéndose de sus competencias como la inteligencia, el esfuerzo y el carisma deberá poder mejorar su autoconciencia y la de sus colaboradores para crear una coalición capaz de transformar su entorno hospitalario, y así integrar el

Unidad de Práctica Integrada en Falla Intestinal, Hospital San Javier Guadalajara, México. ExPresidente de la FELANPE. proceso de atención nutricional dentro de la Cultura de Seguridad Institucional ${ }^{(10)}$ cuyos objetivos son: la identificación y control de los riesgos que pueden causar daño nutricional a los pacientes, la prevención del daño y el proveer un ambiente seguro para proporcionar la alimentación óptima en todos los tiempos y bajo todas las circunstancias.

Por otro lado, la cultura de la seguridad es materia de la ética y está ligada a la eficiencia, ya que un médico seguro no es aquél más competente en una destreza, sino aquél que es más honesto en reconocer sus propios límites de competencias y habilidades y que tiene el valor suficiente para solicitar ayuda y está comprometido para aprender más allá de su límite, especialmente en el área nutricional ${ }^{(11)}$.

Dicho cambio cultural es una precondición para la acción que conducirá a una política para mejorar el proceso de la atención nutricional del paciente con una política de medición de resultados y con ellos la capacidad para actuar en el campo político ${ }^{(12)}$.

Sin duda, la confianza es la piedra angular en la relación médico-paciente ${ }^{(13)}$. El concepto de vulnerabilidad es particularmente relevante cuando el estado de dependencia está relacionada con la enfermedad; por tanto, la persona que está enferma confia en que los

*Correspondencia: Humberto Arenas Márquez arenas50@hotmail.com 
profesionales de la medicina le proporcionemos una atención responsable y digna al reconocer su integridad para mantener su salud y coordinar la atención centrada en las necesidades del paciente para que así los pacientes perciban que están protejidos cuando están más vulnerables.

Aunque tenemos avances significativos en las ciencias médicas, las prácticas no han evolucionado. Para lograr las metas de la Declaración de Cartagena necesitamos una meta unificada que permita alinear todas las acciones.

Un objetivo que ha sido discutido en debates en la atención para la salud y que puede ser definido y medido es el añadir valor a los sistemas. Porter ha desarrollado la teoría del valor donde el centro de la atención, y por ende del valor, son los pacientes. El valor en salud se define como el resultado alcanzado por cada peso invertido y es creado en un ciclo completo de cuidados de la condición de salud de la persona. El valor puede ser medido utilizando herramientas científicas ${ }^{(14,15)}$.

La responsabilidad compartida se logrará mediante el desarrollo de unidades de práctica integrada. Estos sistemas integrados de atención reemplazarían nuestro cultura individualista y autónoma, en los que departamentos y especialidades específicos trabajan de forma independiente y se concentran en proporcionar una gama limitada de intervenciones.

\section{CONCLUSIONES}

Para lograr las metas de la Declaración de Cartagena es fundamental que se produzca un cambio cultural en los médicos. Los sistemas deberán invertir en un modelo integral de atención para la salud que incluya la óptima nutrición. La investigación de la actitud de los médicos a través de la medición de sus resultados y costos, así como la satisfacción del paciente permitirá identificar equipos de atención interdisciplinaria que den valor al paciente al incluir el proceso nutricional en la cultura institucional. Dicho cambio cultural conducirá a lograr una atención de calidad, eficiencia operacional y salud financiera para la institución y los proveedores de atención en beneficio del paciente ${ }^{(16)}$. 


\title{
La Declaración de Cartagena: el papel del farmacéutico
}

\author{
The Cartagena Declaration: the role of the pharmacist \\ A Declaração de Cartagena: o papel do farmacêutico
}

Prof. Gil Hardy, Ph.D, FRSC, FASPEN ${ }^{1 *}$

\begin{abstract}
La Declaración de Cartagena ${ }^{(9)}$ sobre el derecho al cuidado nutricional describe 13 principios importantes, incluido el hecho de que este debe ser un proceso continuo que abarque: detectar, nutrir, vigilar. La alimentación por vía oral, nutrición enteral (NE) o la nutrición parenteral (NP) se consideran una intervención médica, que como cualquier terapia, tiene efectos secundarios, riesgos y beneficios; y por lo tanto, debe ser monitoreada y documentada. Además, los productos nutricionales utilizados para la NP se clasifican como medicamentos farmacéuticos. El Principio \# 4 de la Declaración reconoce $\ll$ El enfoque interdisciplinario del cuidado nutricional». Recomienda específicamente que el cuidado nutricional (en los hospitales) sea realizado por equipos interdisciplinarios, es decir, equipos interdisciplinarios de soporte o terapia nutricional (Nutrition Support Team, NST, por sus siglas en inglés) que debe incluir, como mínimo, profesionales de la nutrición (dietistas), enfermeros, médicos y farmacéuticos.
\end{abstract}

\section{ROL TÉCNICO}

El proceso de cuidado nutricional implica varias etapas. Los farmacéuticos están bien posicionados y profesionalmente calificados para desempeñar un papel importante en cada una de estas fases, utilizando el conocimiento y las habilidades técnicas desarrolladas durante su formación profesional y experiencia de posgrado. En muchos lugares, la formulación y composición de las mezclas de NP sigue siendo la piedra angular de este papel técnico del farmacéutico. Desde el inicio de la NP, los farmacéuticos han tenido la res-

Profesor Emérito de Nutrición Clínica. College of Health, Massey University, Auckland, Nueva Zelanda. ponsabilidad de su preparación. Como expertos en farmacología, microbiología, asepsia y compatibilidad / estabilidad farmacéutica, los farmacéuticos han contribuido significativamente en el uso exitoso de la terapia nutricional, al formular regímenes estables de NP que satisfacen con seguridad las necesidades individuales de cada paciente. Es bien sabido que la administración segura es clave para una terapia nutricional eficaz. Muchos componentes y aditivos de la NP son excelentes medios de crecimiento para bacterias y hongos, pero cualquier riesgo de este tipo puede minimizarse cuando los procesos son supervisados por farmacéuticos. Se puede garantizar una calidad óptima cuando el personal técnico competente prepara las mezclas de $\mathrm{NP}$ en instalaciones asépticas apropiadas y monitoreadas en forma rutinaria.

En la actualidad se dispone de una gran cantidad de datos de investigación, generados con frecuencia por farmacéuticos hospitalarios, académicos y de la industria, para facilitar los juicios sobre formulaciones estables y seguras. La ausencia de insumos farmacéuticos adecuados y el desconocimiento de todos los problemas que influyen en la estabilidad de la mezclas parenterales puede conducir a consecuencias graves, incluso fatales ${ }^{(17)}$. Los programas de capacitación y entrenamiento para farmacéuticos y técnicos de farmacia deben involucrar la comprensión de los principios y limitaciones de las técnicas asépticas, superando la creencia de que cualquier operación realizada bajo flujo laminar automáticamente da como resultado un producto estéril o que una buena validación anula una instalación mal diseñada o mal auditada. Puede haber

*Correspondencia: Gil Hardy gil.hardy50@gmail.com 
un riesgo estadístico de producir bolsas de $\mathrm{NP}$ «contaminadas» en una unidad aséptica mal diseñada y no controlada, operada por personal con capacitación inadecuada. Por otro lado, se puede garantizar la seguridad y la calidad si los farmacéuticos adecuadamente capacitados, que conocen por completo los procesos técnicos involucrados, pueden anular los riesgos microbiológicos potenciales siguiendo los procedimientos operativos estándar y asumiendo la responsabilidad profesional de sus acciones.

\section{ROL CLÍNICO}

La participación del farmacéutico en rondas diarias con los equipos de soporte nutricional proporciona un foro útil para ayudar a identificar eventos inesperados, como fiebre, evitar la eliminación innecesaria de líneas centrales y mejorar la probabilidad de identificar rápidamente las causas de tales eventos que pueden amenazar los resultados del paciente. Su conocimiento especializado sobre los límites de la compatibilidad química dentro de los cuales se puede formular la NP es invaluable ${ }^{(18)}$.

El farmacéutico también puede revisar la administración concomitante de otros medicamentos y asesorar al personal de la sala sobre los aspectos farmacéuticos de la técnica de administración segura. El conocimiento del farmacéutico de la farmacocinética de los medicamentos, las interacciones entre medicamentos y nutrientes (IMN), la estabilidad y la compatibilidad garantizarán que se pueda prescribir la mezcla de NP más conveniente y segura para el paciente ${ }^{(19)}$. Conocer los productos disponibles en el hospital y sus costos también puede garantizar la rentabilidad de la terapia nutricional con NP. La colaboración interdisciplinaria durante la selección y monitorización del paciente, la colocación y cuidado del catéter, la prescripción y preparación de la NP es esencial. En todas estas acciones hay elementos de riesgo y de beneficio para los pacientes. Si se encuentra el equilibrio correcto, entonces los llamados «peligros de la NP» pueden minimizarse o incluso eliminarse. Involucrar a los farmacéuticos en los procesos de evaluación y prescripción de pacientes, permite abordar estos importantes problemas clínicos.

\section{BOLSAS MULTICÁMARA}

Uno de los desarrollos de la NP más significativos en las últimas décadas ha sido el cambio de sistema de botellas múltiples a bolsas individuales que contienen todos los nutrientes que se deben administrar a diario. Muchos regímenes de NP todavía se combinan en bol- sas individuales en unidades asépticas hospitalarias, pero últimamente la industria ha podido proporcionar una amplia variedad de regímenes en bolsas de 2 y 3 cámaras (Multi-Chamber Bags, MCB, por sus siglas en inglés). Los beneficios de administrar NP en una sola bolsa multicámara han sido bien documentados y aceptados como el medio óptimo para una administración segura. Una consecuencia desafortunada de la disponibilidad de MCB con una vida útil larga junto con las crecientes presiones regulatorias sobre buenas prácticas de manufactura, etc., ha sido el abandono de la preparación de la NP por parte de muchas farmacias hospitalarias y el cierre de sus unidades asépticas. Sin embargo, las MCB suministradas por el fabricante no son regímenes «todo en uno»y aún requieren la adición aséptica diaria de micronutrientes. Por lo tanto, los farmacéuticos deben estar atentos y asegurarse de que todas las adiciones a la NP se hagan en forma aséptica, bajo condiciones farmacéuticamente controladas.

\section{FARMACONUTRICIÓN}

Con el continuo interés clínico en los 'nutracéuticos' para la inmuno / farmaconutrición, es probable que se centre cada vez más en la administración parenteral de aminoácidos específicos, como glutamina, arginina, nuevas fuentes de lípidos, como aceites de pescado y los aceites de oliva, oligoelementos individuales y vitaminas antioxidantes, en concentraciones mayores que las RDA (Recommended Dietary Allowance). La Asociación Nutracéutica Americana definió los «nutracéuticos» como «sustancias dietéticas naturales en formas farmacéuticas» ${ }^{(20)}$. Esta definición ciertamente abarca componentes de la NP y farmaconutrientes, pero se ha estimado que $75 \%$ de los usuarios domiciliarios de suplementos nutracéuticos no informan a su médico o al equipo de soporte nutricional y pueden no considerarlos como «medicamentos». De hecho, muchos nutracéuticos son medicamentos potentes con algunos beneficios para la salud, pero pueden tener efectos potencialmente adversos al interactuar con la nutrición parenteral o enteral. Se conocen las interacciones entre tiamina / bisulfito, cisteína / cobre, selenito / ácido ascórbico, lípidos / peróxidos. Sabemos que la glutamina afecta la farmacocinética del metotrexato en pacientes con cáncer y la metionina aumenta la incidencia de oxoprolinuria en mujeres con dietas bajas en proteínas. El aumento de las solicitudes clínicas para complementar los regímenes de NE y NP con dosis farmacológicas de estos nutracéuticos para el tratamiento 
de enfermedades específicas requerirá una mayor participación del farmacéutico para la preparación aséptica de los regímenes de farmaconutrición para pacientes específicos. ¡No menos!

\section{ROL DE ENSEÑANZA}

La experiencia y capacitación del farmacéutico en farmacocinética, inmunonutrición y microbiología los convierte en una fuente principal de conocimiento, asesoramiento y educación para profesionales de la salud y pacientes con NP. Como miembro clave dentro del equipo de soporte nutricional interdisciplinario, el farmacéutico puede garantizar que sus colegas desarrollen una mejor conciencia de los riesgos potenciales de los medicamentos en las etapas de evaluación del paciente. La experiencia en asesoría sobre medicamentos permite al farmacéutico educar y orientar a los pacientes sobre la preparación, el almacenamiento y la administración de NP en el hogar (Home Parenteral Nutrition, HPN, por sus siglas en inglés). La educación y la capacitación continua de otros miembros del equipo de soporte nutricional y pacientes con HPN en técnicas asépticas para manejar la NP y la administración conjunta de otros medicamentos es una responsabilidad clave del farmacéutico. La centralización de todos los registros derivados de la prescripción y del monitoreo de pacientes en la farmacia, puede constituir una parte esencial del plan de atención del paciente con nutrición parenteral y facilitará auditorías periódicas para mejorar constantemente el desempeño del equipo en la lucha contra la desnutrición relacionada con la enfermedad, como defiende la Declaración de Cartagena. 


\title{
El derecho al cuidado nutricional y la lucha contra la malnutrición. Desde la óptica del Nutricionista
}

\author{
The right to nutritional care and the fight against malnutrition. From the \\ Nutritionist's point of view \\ O direito ao cuidado nutricional e a luta contra a desnutrição. Do ponto de vista do \\ Nutricionista
}

María Alejandra Texeira ND., MSc. ${ }^{*}$, Yadira Cortes ND., Ph.D. ${ }^{2}$, Edna Nava ${ }^{3}$, Laura Joy ND., Ph.D. ${ }^{4}$

La reflexión enfocada en la Declaración de Cartagena ${ }^{(9)}$ desde el Comité de Nutricionistas de la Federación Latinoamericana de Terapia Nutricional, Nutrición Clínica y Metabolismo (FELANPE) nos lleva a preguntarnos iCuál es nuestra responsabilidad como profesionales y como colectivo en relación con estos derechos?

La respuesta sin duda es que como profesionales y como colectivo tenemos la responsabilidad de contribuir a la promoción del derecho al cuidado nutricional y lucha contra la malnutrición, y al avance en temas de educación e investigación en nutrición clínica, para que sean una realidad y no solo enunciados teóricos de principios que no se ponen en práctica.

$\mathrm{Si}$ bien los enunciados de principios y las normas son importantes, sin los sentimientos, las actitudes y la convicción interna, no obtendremos los resultados esperados. Como profesionales de la nutrición pensamos que no basta con conocer la Declaración, sino que, es necesario asumir el espíritu de la misma y llevarla a cabo.

Como colectivo es nuestra obligación defender este derecho no solo cumpliéndolo desde el ejercicio de nuestra práctica diaria, sino que, además debemos velar para que se respete y se cumpla evitando caer en una situación de complicidad o de falta por omisión.

Banco de Leche Humana del Hospital Regional de Salto. Salto, Uruguay. Directora del Comité de Nutrición de la FELANPE 2019 - 2020.

2 Departamento de Nutrición y Bioquímica, Pontificia Universidad Javeriana, Bogotá, D.C., Colombia. Comité de Nutrición de la FELANPE, Región Centro, 2019 - 2020.
JL Aranguren plantea la siguiente pregunta ipuede ser considerado como verdaderamente bueno el hombre que acepta, cuando menos con su pasividad y con su silencio, una situación social injusta? ${ }^{(21)}$

Al trasladar el interrogante de Aranguren al contexto de la Declaración de Cartagena nos preguntamos: ¿puede ser considerado bueno el Nutricionista que acepta, cuando menos con su pasividad, la violación del derecho del cuidado nutricional y todo lo que esto implica?

Es responsabilidad del Nutricionista participar en la formulación e implementación de políticas para el cumplimiento positivo de los postulados de la Declaración de Cartagena, analizar las estructuras y políticas gubernamentales y, a la vez, llevar a cabo investigaciones en nutrición y salud que aporten datos para la elaboración de estas políticas.

Demostrar, mediante la investigación clínica, que las intervenciones del profesional de la nutrición resuelven problemas, ayudan a las personas a tener pleno goce de sus derechos a un estado nutricional y de salud adecuados, es un compromiso individual como profesionales y además un tema de responsabilidad gremial y social.

Los Nutricionistas debemos realizar una práctica que priorice la conservación de los derechos de las per-

\footnotetext{
3 Universidad Autónoma de Nuevo León, México. Comité de Nutrición de la FELANPE, Región Norte, 2019 - 2020.

4 Instituto Nacional de Cáncer, Paraguay. Comité de Nutrición de la FELANPE, Región Sur, 2019 - 2020.
} 
sonas, que promueva el reconocimiento de la nutrición y la salud como un derecho humano, abogando por las personas que están bajo nuestro cuidado.

Pensamos que impulsados por la Declaración de Cartagena debemos conformar grupos académicos y de estudio de ética, con docentes, investigadores y clínicos, con el propósito de lograr un mayor acercamiento entre la teoría y la práctica, asumiendo el compromiso de ser no solo un buen Nutricionista, sino un Nutricionista preparado, actualizado, ético, con conciencia gremial y profesionalismo. 


\title{
Declaración Internacional sobre el derecho al cuidado nutricional y la lucha contra la malnutrición. Desde la perspectiva de Enfermería
}

\author{
International declaration on the rigth to nutritional care and the fight against \\ the malnutrition. From the Nursing perspective \\ Declaração internacional sobre o direito aos cuidados nutricionais e o combate contra à \\ desnutrição. Na perspetiva da Enfermagem
}

María Isabel Pedreira de Freitas, Ph.D. ${ }^{1 *}$

En mayo de 2019, la Federación Latinoamericana de Terapia Nutricional, Nutrición Clínica y Metabolismo (FELANPE), en asamblea general, aprobó la Declaración internacional sobre el derecho al cuidado nutricional y la lucha contra la malnutrición, llamada Declaración Cartagena.

El personal de Enfermería es testigo a diario de pacientes que se desnutren durante su hospitalización o que llegan a las instituciones de salud en un estado de desnutrición lamentable ${ }^{(22)}$. Esta situación aflige en gran medida a personas que permanecen las 24 horas del día al lado de quienes buscan respuesta a sus necesidades de salud. Se conocen publicaciones de estudios basados en evidencia ${ }^{(23)}$, en los que pacientes que permanecen en ayuno para cirugía, por un tiempo superior a lo recomendado, ponen en riesgo su recuperación ${ }^{(24)}$. Sin embargo, en muchas instituciones de salud, todavía hay resistencia a hacer modificaciones a esta práctica que puede llegar a comprometer la vida del paciente quirúrgico.

Como enfermeros ¿qué tenemos que ver y hacer con esta Declaración, si sabemos que junto a los pacientes y sus familiares, actuamos en el mismo campo de los médicos?

\section{iTENEMOS MUCHOS QUE VER Y HACER!}

Es una fortuna saber que Estados de todo el mundo, presididos por el doctor Tedros Ghebreyesus, Director General de la Organización Mundial de la Salud,

Ex Presidente del Comité de Enfermería de la FELANPE
OMS, y el Consejo Internacional de Enfermería, CIE, durante la inauguración de la 72 Asamblea Mundial de la Salud, establecieron 2020 como el Año de la Enfermera y de la Partera (matrona, comadrona), dando origen a Nursing Now.

Esta campaña afirma que "Si bien es cierto que la OMS reconoce el papel crucial que los enfermeros realizan a diario, 2020 será dedicado a destacar los enormes sacrificios y contribuciones de las enfermeras y parteras, y... busca capacitar a los enfermeros para que ocupen su lugar en el corazón de los desafíos de la salud del Siglo XXI y maximicen su contribución para alcanzar la Cobertura Universal de la Salud"(25)

Caminar junto a los profesionales de la salud que apoyan la Declaración de Cartagena, significa que Enfermería puede optimizar cada paso en dirección a los retos que la atención de la salud exigen, para que esta se haga teniendo en cuenta que "promover el desarrollo del cuidado nutricional en el ámbito clínico que permita que todas las personas enfermas reciban terapia nutricional en condiciones de dignidad"(9) es uno de los objetivos de la Declaración.

¿Quién mejor que el equipo de enfermería que acompaña al paciente en su hospitalización en los diferentes momentos en los que sus condiciones clínicas cambian, provocando modificaciones en las conductas terapéuticas que afectarán su recuperación y su estado general?

Los enfermeros están en capacidad de observar y actuar sobre los "factores tan diversos como educación,

*Correspondencia: María Isabel Pedreira de Freitas beloca4@yahoo.com.br 
estado económico, capital social y ambiente físico que afectan profundamente la salud y el bienestar del individuo y de las poblaciones" como dice uno de los principios del documento Nursing Now ${ }^{(25)}$.

$\mathrm{Al}$ concebir el cuidado nutricional como "un proceso continuo que consta de diversas etapas las cuales se pueden resumir en: 1. Detectar, 2. Nutrir y 3. Vigilar", como lo plantea la Declaración de Cartagena, el profesional de la salud que está más próximo al paciente y puede actuar de manera inmediata al detectar las alteraciones presentes en la evolución del paciente y en consecuencia proporcionar sus cuidados, es el Enfermero. Este actúa en todo el proceso de la asistencia con un fuerte abordaje de enseñanza de los cuidados postoperatorios, directamente asociada a una mejor recuperación del paciente, así como la evaluación y monitoreo continuos, están muy bien definidos en el papel del enfermero $^{(26)}$.

Como dice la Declaración de Cartagena, "la terapia nutricional se debe monitorear y documentar. El monitoreo busca verificar que se cumplan las distintas dimensiones de la terapia nutricional y prevenir los efectos secundarios. La documentación sirve para seguir y evaluar la continuidad de la terapia de cada paciente y garantizar la calidad" ${ }^{\prime(9)}$. Será el liderazgo del enfermero en la asistencia lo que favorecerá el cumplimiento de este registro y el monitoreo de las complicaciones que ocurren en el proceso de hospitalización del paciente. Son estas acciones, a partir de los resultados obtenidos en las experiencias exitosas vividas en la cotidianidad con los pacientes, ciudadanos y profesionales de la salud, las que hicieron posible las propuestas registradas en el documento Nursing Now y en la Declaración de Cartagena que gratamente caminan hacia un mismo objetivo, hombro a hombro, optimizando los resultados que se deben alcanzar.

Tener conciencia de que la "La investigación en nutrición clínica es un pilar para el cumplimiento del derecho al cuidado nutricional y la lucha contra la malnutrición" hace necesario fomentar "la enseñanza de la nutrición clínica en las carreras de la salud (medicina, nutrición, enfermería, y farmacia, entre otras)."(9) Por lo tanto, es fundamental la actuación interdisciplinar en la formación de los alumnos en las áreas de la salud, desde el inicio de la formación curricular, para lograr una interacción efectiva y coherente con el objeto de obtener los mejores resultados en la práctica clínica.

Para que el paciente atendido pueda ser alimentado y estar nutrido de forma adecuada, es necesario que cada persona realice acciones concretas. Los profesionales de la salud deben actuar conociendo el nivel cultural de cada paciente y su familia, con el fin de empoderarlos para que puedan dar continuidad al proceso de recuperación de la salud en su domicilio. Podrán tomar las mejores decisiones basadas en la libertad que proporciona el conocimiento, identificando lo que es mejor para él y para su salud. Esa libertad puede ser alcanzada cuando incorpora la capacitación y enseñanza que el enfermero elaboró con su equipo y transmitió al paciente durante su periodo de hospitalización, optimizando de esta manera los resultados obtenidos durante el proceso de enseñanza aprendizaje ${ }^{(27)}$.

La Declaración de Cartagena viene a fortalecer las acciones que los equipos de salud deben desarrollar junto a las personas que buscan mejorar su salud cuando esta se encuentra comprometida.

\section{Financiación}

El presente artículo no tuvo financiación.

\section{Conflicto de intereses}

Declaro no tener conflicto de intereses

\section{Declaracion de autoria}

SE diseñó el artículo. Todos los autores participaron por igual y validaron la versión final.

\section{Referencias bibliográficas}

1. Barrocas A. Demonstrating the Value of the Nutrition Support Team to the C-Suite in a Value-Based Environment: Rise or Demise of Nutrition Support Teams? Nutr Clin Pract. 2019;34:806-21.

2. Tamayo y Tamayo M. El Método, la interdisciplinariedad y la universidad. Universidad ICESI, 1995. (Consultado el 26 de octubre de 2019). Disponible en: https://books.google.pt > books

3. McCauley SM, Barrocas A, Malone A. Malnutrition quality improvement initiative yields value for interdisciplinary patient care and clinical nutrition practice. J Acad Nutr Diet. 2019;119(9):S1-S72.

4. Correia MI, Hegari RA. Addressing Disease -Related Malnutrition in Healthcare: A Latin-American Perspective. JPEN J Parenter Enter Nutr. 2016;40(3):319-25.

5. Arenas D, Plascencia A, Ornelas D, Arenas H. Hospital Malnutrition Related to Fasting and Underfeeding Is It an Ethical Issue? Nutr Clin Pract. 2016;31(3):316-24.

6. Yeh DD, Fuentes E, Quraishi SA, Cropano C, Kaafarani H MA, et al. Adequate Nutrition May Get You Home: Effect of Caloric/ Protein Deficits on the Discharge Destination of 
Critically Ill Surgical. Patients. JPEN J Parenter Enteral Nutr. 2016;40(1):37-44.

7. Malone A. Addressing Hospital Malnutrition- The Time Is Now! JPEN J Parenter Enteral Nutr. 2013;37:439-40.

8. Tapenden KA. The value of Nutrition Intervention. JPEN J Parenter Enteral Nutr. 2013;37(2):160.

9. Cardenas D, Bermúdez CH, Echeverri S, Perez A, Puentes M, Lopez M, et al. Declaración de Cartagena. Declaración Internacional sobre el Derecho al Cuidado Nutricional y la Lucha contra la Malnutrición. Nutr Hosp. 2019;36(4):97498. http://dx.doi.org/10.20960/nh.02701.

10. McLean S. Transformation: A New Kind of Academic Health Center and the Pursuit of High Self-Mastery. J Am Coll Surg. 2016;222(4): 337-46.

11. Thurunavukarasu P. Do more requirements make better surgeons? External vs. Internal motivators: Against increasing requirements. Bull Am Coll Surg. 2011; 96(8):50-1.

12. Leape L, Berwick D, Clancy C, Conway J. Gluck P, Guest J, et al. Transforming healthcare: a safety imperative. Qual Saf Health Care. 2009; 18:424-8.

13. Pellegrini C. Trust: The Keystone of the Patient-Physician Relationship. J Am Coll Surg. 2017;224(2):95-102.

14. Porter ME, Teisberg EO. Redefining Healthcare: Value-based Competition on Results. Boston, MA: Harvard Business School Press; 2006.

15. Hoyt DB.Looking forward. Bull Am Coll Surg. 2011;96(12):46.

16. Knight A. Pride and Joy. Linney Group Ltd 2014. Chapter Five. Crocodiles. p. 21-31.
17. Allwood M, Hardy G, Sizer T. Roles and functions of the pharmacist in the nutrition support team. Nutrition. 1996;12(1): 63-4.

18. Allwood MC, Ronchera-Oms CL, Sizer T, McElroy B and Hardy G. From pharmaceutics to pharmaceutical care in nutritional support. Clin Nutr. 1995:14 (1);1-3.

19. Ayers P, Boullata J, Sacks G. Parenteral Nutrition Safety: The Story Continues. Nutr Clin Pract. 2018;33(1): 46-52.

20. Mannion M. Nutraceutical revolution continues at Foundation for Innovation in Medicine Conference. Am J Nat Med. 1998;5:30-3.

21. Aranguren JL. Ética. Barcelona: Altaya; 1994. p. 38.

22. Correia MITD, Campos AC; ELAN Cooperative Study. Prevalence of hospital malnutrition in Latin America: the multicenter ELAN study. Nutrition. 2003; 19(10):823-5.

23. Correia MITD, Silva RG. Paradigms and evidence of perioperative nutrition. Rev. Col. Bras. Cir. 2005; 32(6):342-347. http://dx.doi.org/10.1590/S0100-69912005000600012

24. Kehlet H, Wilmore DW. Multimodal strategies to improve surgical outcome. Am J Surg. 2002;183(6):630-41.

25. World Health Organization. Nursing Now campaign: empowering nurses to improve global health. [internet] 27.02.2018. (Consultado el 30 de octubre 2019) Disponible en: https:// www.who.int/hrh/news/2018/nursing_now_campaign/en/

26. Nursing Now - Brasil. Onde há pesquisa há enfermagem. [Internet] (Consultado el 30 de octubre 2019). Disponible en: http://nursingnowbr.org/principios-internacionais

27. Crosson JA. Enhanced Recovery After Surgery - The Importance of the perianesthesia Nurse on Program Success. J Perianest Nurs. 2018; 33(4):366-74. 